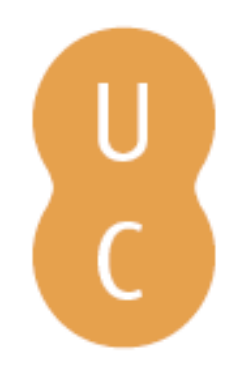

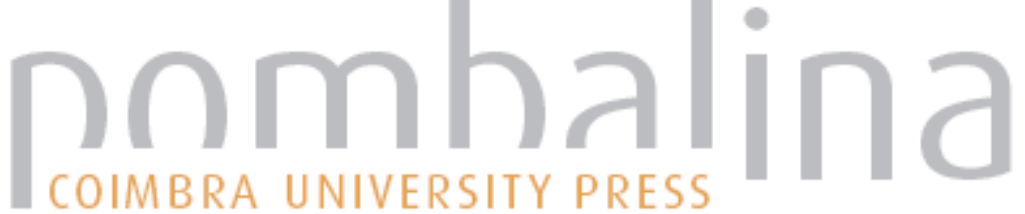

\section{Télévision et Citoyenneté}

\author{
Autor(es): Nel, Noël
}

Publicado por: Imprensa da Universidade de Coimbra

URL

persistente:

URI:http://hdl.handle.net/10316.2/36652

DOI:

DOI:http://dx.doi.org/10.14195/978-989-26-0873-0_13

Accessed : $\quad$ 26-Apr-2023 11:59:20

A navegação consulta e descarregamento dos títulos inseridos nas Bibliotecas Digitais UC Digitalis, UC Pombalina e UC Impactum, pressupõem a aceitação plena e sem reservas dos Termos e Condições de Uso destas Bibliotecas Digitais, disponíveis em https://digitalis.uc.pt/pt-pt/termos.

Conforme exposto nos referidos Termos e Condições de Uso, o descarregamento de títulos de acesso restrito requer uma licença válida de autorização devendo o utilizador aceder ao(s) documento(s) a partir de um endereço de IP da instituição detentora da supramencionada licença.

Ao utilizador é apenas permitido o descarregamento para uso pessoal, pelo que o emprego do(s) título(s) descarregado(s) para outro fim, designadamente comercial, carece de autorização do respetivo autor ou editor da obra.

Na medida em que todas as obras da UC Digitalis se encontram protegidas pelo Código do Direito de Autor e Direitos Conexos e demais legislação aplicável, toda a cópia, parcial ou total, deste documento, nos casos em que é legalmente admitida, deverá conter ou fazer-se acompanhar por este aviso.

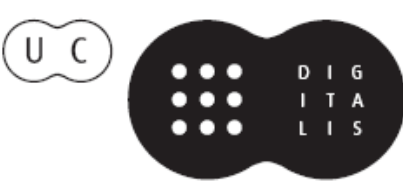


www.uc.pt/ imprensa_uc CONTACTO imprensa@uc.pt VENDAS ONLINE http://livrariadaimprensa.uc.pt JANEIRO 2015
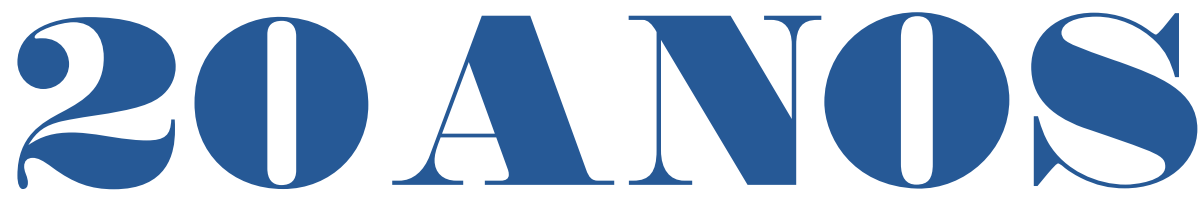

\section{DE JORNALISMO CONTRA A INDIFERENÇA}

TEXTOS DE

Marc Lits, Adriano Duarte Rodrigues, Tito Cardoso e Cunha, José Augusto Mourão, Alberto Pena Rodríguez, Maria Augusta Babo, Daniel Cronu, João Pissarra Esteves, Gilles Gauthier, Heloísa Paulo e Luís Reis Torgal, Alfredo Barroso, António Fidalgo, Nöel Nel, João de Almeida Santos, Juan Luis Cebrián, António Dias Figueiredo, Marina Themudo, Jorge Sampaio, Nelson Traquina, Mário Soares
( livro que agora se apresenta, nasce de dois desígnios fundamentais: por um lado, celebrar duas décadas de ensino do Jornalismo na Universidade de Coimbra e, por outro, partilhar com um público mais alargado um conjunto de reflexões sobre os media, o jornalismo, a comunicação e o espaço público.

Se o ensino superior do Jornalismo em Portugal, relativamente tardio em relação ao resto da Europa, deu os seus primeiros passos no fim dos anos 70 do século passado, ele aparece apenas duas décadas depois na academia coimbrã. Contudo, esta foi, no contexto nacional, a primeira licenciatura em Jornalismo, distinguindo-se, quer em título, quer em objetivos, das licenciaturas então existentes no país. A criação de uma Licenciatura em Jornalismo na Universidade de Coimbra, em 1993-1994, foi, por si, um acontecimento. Com efeito foi necessário que reitor, professores e jornalistas ousassem atualizar a oferta curricular da Faculdade de Letras, oferecendo um curso há muito desejado pela sociedade e pelo mercado, embora desconsiderado por alguns setores da academia. Correndo o risco de omitir alguém, a quem antecipadamente pedimos desculpa, não podemos deixar de recordar os esforços dos jornalistas João Mesquita, João Fonseca, em representação
Todas as gerações, sem dúvida, se julgan para refazer o mundo. A minha sabe, nc que não poderá refazê-lo. A sua tarefa é tc

Consiste em impedir que se desfaça, $p$ unicamente das suas negações A. Camus, Discursos da Suécia (1957)

do Sindicato dos Jornalistas, e de Jorge Castilho, a quem mais tarde se viria associar o nome de Mário Martins, bem como o do então Reitor da Universidade de Coimbra Rui Alarcão, e dos professores João Roque e Luís Reis Torgal. Entre 1993 e 1996, a Licenciatura em Jornalismo funcionou com um Secretariado, que teve um papel executivo e científico nos primeiros tempos do curso na FLUC. Presidido pelo Presidente do Conselho Científico Ludwig Scheidl, este 


\title{
Télévision et Citoyenneté
}

\author{
Noël Nel \\ Université de Metz
}

Objet hypercomplexe et ouvert, car placé en situation d'intermédiation, au carrefour de phénomènes économiques, sociaux, culturels, politiques, la télévision appelle une approche minutieuse, loin des réductions simplistes dont on ne cesse de la gratifier généreusement. Certaines notions et certains concepts sont alors indispensables pour cerner cet objet beaucoup plus subtil qu'il n'y paraît.

Sans aller jusqu'à affirmer l'existence de médiasphères ${ }^{1}$ gouvernant la vie sociale au plan mondial et imposant aux territoires sous leur juridiction d'implacables écosystèmes; sans verser dans les raccourcis "totalisants" de l'homme unidimensionnel (Marcuse), du tribalisme planétaire (Mac Luhan) ou du simulacre sidérant (Baudrillard); sans brandir le scénario tragique (Virilio) contre le scénario béat (Quéau) ou vice versa, il nous faut bien constater, à l'instar d'Eliseo Véron ${ }^{2}$ que nous habitons des sociétés occidentales en voie de médiatisation. Il s'agit de sociétés où des pratiques sociales (des modalités de fonctionnement institutionnel, des mécanismes de prise de décision, des habitudes de consommation, des comportements plus ou moins ritualisés, etc.) se transforment du fait qu'il y a des médias. Dans ces types de sociétés, comment prendre la juste mesure du rôle tenu par la télévision, au regard de la vie démocratique?

Ni appareil idéologique d'Etat, ni simple machine de communication, d'organisation et de gestion, la télévision est, au sens plein du terme, un média, c'est-à-dire une institution puissante marquée par la pluralité fonctionnelle. Elle repose sur des fonctions originelles et permanentes, des fonctions plus récentes et non prévues au départ, des fonctions plus ou moins remplies en même temps par d'autres institutions sociales. Elle est tout à la fois média d'information, média de distraction, média de culture, média de liaison sociale. C'est ce dernier aspect qui sera frontalement abordé dans ce qui suit. Mais pour en parler avec le maximum de pertinence, il est préalablement nécessaire de préciser ce que l'on devrait entendre par le terme de télévision.

\section{La télévision comme objet hypercomplexe}

On peut aujourd'hui affirmer que l'oubli de penser la médiation dans l'action du média télévision a favorisé jadis l'impérialisme du paradigme 
des effets puissants et directs, ces théories simplistes du conditionnement et de la manipulation permanente. Si donc l'on veut avoir une idée plus juste du fonctionnement de la télévision, notamment de la télévision de la fin du vingtième siècle, il faut commencer par prendre le média pour une institution de médiation.

\subsection{La télévision comme média}

Placé par nature en situation intermédiaire, le média télévision nous oblige à considérer deux niveaux étroitement interdépendants, que J-M Salaün ${ }^{3}$ nomme le niveau sociétal de la régulation sociale générale - le média dans son environnement national et international - et le niveau interne de la régulation spécifique. Ces deux niveaux se superposent-ils? S'emboîtent-ils? Une autonomie relative y est-elle possible? Autant de questions qui réclameraient en réponse des inventaires précis!

La médiation télévisuelle est une opération d'interconnexion entre de multiples séries, matérielles et techniques, institutionnelles, idéologiques et politiques, humaines, économiques, symboliques. Elle s'inscrit alors dans une théorie de l'action qu'il ne faut pas penser sur le seul mode simplificateur de la rationalité stratégique, mais comme le réseau complexe de multiples logiques sociales ${ }^{4}$. Portées par un agir communicationnel dont il sera question plus loin, ces logiques à la fois concurrentes et complémentaires développent des régimes d'action qui tentent de les structurer pour leur donner cohérence et synergie. Et, si l'on veut bien admettre qu'un média est toujours un réseau de liaisons, transmissions ou circulations entre un monde matériel et un monde symbolique, on pourra alors distinguer un régime d'action matérielle - régime technique et économique - et un régime d'action symbolique, que j'ai proposé de nommer régime scopiques et qui peut à son tour se subdiviser en régime cognitif d'intelligibilité, régime perceptif de visibilité-audibilité, régime passionnel d'affectivité5. Ces deux régimes d'actions co-occurrentes font de la médiation un procès de complexification.

\subsection{Le régime d'action matérielle}

A ce premier niveau, la médiation est à considérer comme facteur d'opacification.

Sur le plan matériel, la médiation comme procès de complexification manifeste l'opacité et la profondeur de la technique télévisuelle. Qu'il s'agisse des secteurs concernés - électronique, télécommunications, infor- 
matique - ou qu'il s'agisse des niveaux à envisager - production, diffusion, réception - la télévision montre la puissance et l'ambivalence d' effets techniques puissants. Prenons les exemples du direct et du réseau.

Spécificité du média, le direct télévisuel se présente comme mise en simultanéité de trois opérations: la mise en cadre, la mise en continuité (découpage-montage) et la mise sur antenne. Il s'accompagne d'un état affectif autonome chez le téléspectateur: le sentiment, plus ou moins fort, de participation immédiate à l'événement retransmis. Il fonctionne donc en opérateur de vérité parce qu'il semble déni de mise en scène, gage d'authenticité, notamment dans la présence d'imperfections techniques. D'un autre côté, la possibilité de simuler ou de parasiter les conditions du direct (transmettre en différé, insérer dans le direct des éléments pré-montés), conduit à brouiller la spécificité dont il s'agit, laquelle tend ainsi à devenir de plus en plus rare.

On peut faire des remarques analogues sur la question du réseau propre à la télévision, réseau physique et figurant à la fois. D'un côté, le réseau de la machine à diffuser sur un large territoire qu'est la télévision structure l'espace-temps du téléspectateur selon l'imaginaire de l'immédiateté, de la simultanéité, de la transparence, de la pluralité et de la stratégie. D'un autre côté, le réseau est frappé d'ambivalence conceptuelle: il est centre (d'émission) et périphérie (de réception); intérieur de studio et extérieur de terrain; continuité d'un flux et discontinuité de programmes d'émissions; visibilité et invisibilité; organisation préalable et réaction à chaud; circulation et contrôle, espace public et espace privé. Il est pluralité de combinaisons ouvrant à d'autres territorialités et laissant imaginer une connectabilité virtuelle, plus limitée cependant que celle du réseau des réseaux, Internet.

Sur le plan économique, la médiation télévisuelle se présente comme opération d'utilisation d'innovations technologiques et industrielles - elles-mêmes au carrefour de plusieurs branches (industrie électrique, télécommunications, spectacle,etc) - au service de la diffusion et de la production d'oeuvres qui deviendront les marchandises d'une industrie. La télévision se nourrit de marchandises culturelles issues d'autres médias (cinéma, photo, livre) en même temps qu'elle propose ses propres produits commerciaux. Et elle tente de donner à des produits issus du secteur de l'information une légitimité ou valeur égale à celle dont bénéficient les oeuvres issues du secteur culturel, ces dernières n'étant pas marquées par un mécanisme d'obsolescence aussi rapide. Ecartelé entre fonction de diffusion et fonction de production, logique éditoriale et logique de flot, secteur commercial et 
secteur culturel, le régime d'action matérielle ne peut donc que tenter d'articuler des logiques complexes.

\subsection{Le régime d'action symbolique}

C'est cependant au plan social, politique et culturel, que la télévision fait régner la complexité la plus grande. Tentent d'en rendre compte les approches qui mettent en avant les notions de champs (Bourdieu), logiques sociales multiples (Miège), "mouvements structurants-structurés" (Bourdieu encore), espace public (Habermas et sa postérité).

Il n'est pas du tout évident que nous puissions inscrire la télévision dans une théorie générale des champs comme "univers extrêmement complexes" qui reposeraient sur des lois générales et immanentes de jeu, des mécanismes universels et structurants de luttes ou rapports de force et des habitus englobant un éthos de valeurs ${ }^{6}$. La première difficulté est en effet de dresser une sorte de cartographie des champs, d'y positionner correctement le champ télévisuel et son réseau d'interconnexions multiples. Bourdieu nous propose de considérer la télévision comme l'un des sous-champs du champ journalistique, les autres étant la presse écrite et la radio. Selon lui, le champ journalistique est "d'une certaine façon englobé dans le champ politique au sein duquel il exerce des effets très puissants" ${ }^{7}$. D'autre part, il est en relation avec les autres champs de la production culturelle

: champs littéraire, artistique, scientifique, juridique,etc. Enfin, ces champs ont en commun d'être "très directement et très étroitement placés sous l'emprise de la sanction du marché et du plébiscite" ". Cette conception du champ journalistique conduit en fait à simplifier le média télévision en le réduisant à la seule fonction journalistique et à la seule finalité commerciale, ce qui ne convient évidemment pas à l'ensemble des chaînes françaises: TF1 s'y retrouve assez bien, mais Arte s'y perd complètement.

Mieux vaut sans doute tenter, à la faveur d'investigations généalogiques ou archéologiques, de repérer les multiples logiques sociales qui se croisent, se combinent ou se combattent à l'intérieur même d'un média comme entre chaînes différentes, selon ce que Miège nomme des " problématiques transversales et partielles”. On sait par exemple que la production industrialisée de l'information et de la culture se partage entre deux modèles génériques, le modèle éditorial (livres, disques, films, vidéo) et le modèle de flot (émissions de radio et de télévision). Or, la télévision de la fin de ce siècle s'engage à présent dans une sorte de statut intermédiaire qui permet à certaines de ses émissions d'information de suivre les règles de fonctionnement des deux 
modèles de référence. Ainsi, certaines émissions de conversation ou de débat répondant à l'actualité d'une époque - B. Pivot recevant à Apostrophes un grand écrivain - sont à présent éditées comme archives mémorables dignes de constituer des documents historiques. En somme, sur le plan symbolique, le média télévision agit dans l'éventail des possibilités que lui offrent les deux modèles économiques disponibles concernant la valorisation des produits culturels.

Mais il nous faut encore et surtout caractériser le mode de symbolisation qui lui est propre. On sait que, lois médiologiques obligent, la télévision articule tous les registres et les médias qui l'ont précédée. On peut alors la décrire sommairement comme l'intégration des médias antérieurs - écriture (édition, presse), parole (radio), images fixes (photo) et animées (film)ainsi que l'hybridation de tous les ordres signifiants: symbolique, iconique et indiciel. Ce dernier surtout, travaillé par la radio selon l'immédiateté du direct et selon le seul canal de la sonorité, se trouve ici déployé dans l'ordre de la visualité. Ainsi s'élabore une autre variété de régime scopique.

On voit donc que, considérer la télévision comme un média porteur d'un régime scopique spécifique, c'est ne pas l'engluer dans la logique réductrice -essentiellement économique - des champs, et donner à la notion de médiation toute son importance.

Il convient à ce moment de rappeler rapidement les caractéristiques essentielles d'un régime scopique, avant de définir les traits propres à celui de la télévision. Engager une critique de la raison iconographique, ce qui est mon objectif, c'est considérer que le champ de la vision est hétérogène et complexe. C'est affirmer que la production-réception des images - picturales, photographiques, cinématographiques, télévisuelles et numériques est gouvernée par des régimes qui définissent les postures d'observation ou regards et les visions de mondes ( contructions de la réalité sociale ou représentations fictionnelles). C'est étudier attentivement les contextes institutionnels des actes de perception et les pratiques sociales de réception que les différents régimes imposent selon le temps, à la faveur de processus perpétuels de variation. Chaque citoyen téléspectateur est donc positionné par le régime scopique télévisuel en un type d'observateur dont les comportements de réception, aux plans cognitif, perceptif, passionnel, varient relativement dans le temps. Cet observateur est à concevoir comme une place, un rôle, un positionnement de sujet dans la trame historique. Au fil du temps, le champ des images et de leurs régimes scopiques semble avoir connu une évolution importante, situable dans la seconde moitié du vingtième siècle: le passage 
d'un méta-régime fondé sur l'iconicité à un autre méta-régime gouverné par la virtualisation. Le premier concerne peinture, photo, cinéma et télévision, tous attirés par la relation de l'image au modèle extérieur,tous occupés par le rapport (vrai)semblable/invraisemblable, possible/impossible, probable/ /improbable, certain/incertain. Le second a bénéficié des technologies informatiques pour numériser l'ensemble des images précédentes et inventer la modélisation, qui donne une visualité aux figures du virtuel. Le premier régime propose l'image-simulacre et l'image-trace d'un référent externe, le second crée l'image-modèle ou image-programme.

La télévision appartient au régime iconique, mais en y occupant une place précise et singulière. Le régime iconique peut être subdivisé en deux domaines: la représentation et la présentation. Bien connu, le champ théorique de la représentation a produit les régimes contemplatifs de la peinture, de la photo et du cinéma. L'observateur-spectateur y est sédentarisé, fixé à une place, soumis à une vision stable de l'image-simulacre. Et celle-ci travaille en permanence un conflit qui l'habite entre renvoi indiciel au réel et économie propre de l'iconicité. Comme le dit D. Chateau: "La grande difficulté du problème réside justement dans le fait que la relation iconique à la fois suppose des conditions indicielles qui permettent l'existence d'un signe susceptible d'être appréhendé comme ressemblant et l'oubli de ces conditions afin que l'attention du récepteur soit concentrée sur la ressemblance et sur elle seule" ".

En apportant le mouvement, le cinéma semble avoir introduit l'indicialité au coeur même de l'iconicité: la scène du film, fictionnelle le plus souvent, paraît se dérouler au présent. Cette qualité particulière de l'iconicité cinématographique produite dans le cadre d' un dispositif d'ancrage de l'observateur à une place imposant la sous-motricité et la sur-perception explique que le cinéma soit synonyme de contemplation. La télévision prolonge ces phénomènes et les porte plus loin, dans le champ de la présentation. Ainsi le direct télévisuel, marqué lui aussi par l' indicialité dynamique, apparaît comme une nouvelle relation à l'espace temps: grâce à la retransmission immédiate (faisceau et réseau), il est une relation à un espace-temps homogène et contemporain, situé au même moment dans l'ailleurs. De plus, la présentation ainsi construite exhibe son site (studio ou terrain de l'information), son maître (présentateur), ses modalités particulières de relation au téléspectateur (le regard-caméra), ses actes de discours spécifiques (présentation verbale et représentation audiovisuelle), ses modes d'activation de l'espace public. Fondé sur le contact, le régime de la présentation se veut phatique. 
On a souvent décrit la présentation télévisuelle, la construction de l'actualité et de l'événementialité en termes de régime de vérité, de discours vériste et de contrat de fiducie ${ }^{10}$. Si ces approches sont pertinentes, il convient cependant de tirer les leçons de quelques affaires récentes: charnier de Timisoara, fausse interview de Fidel Castro par Patrick Poivre d'Arvor, couverture de la guerre du Golfe par CNN. Ces exemples ont le mérite de nous rappeler l'artefactualité de l'image télévisuelle, laquelle est toujours mise en cadre, mise en scène possible, mise en chaîne ou en continuité. Toute retransmission est, en ce sens, production d'artefact. Par ailleurs, dans la présentation télévisuelle se tient une spectralité chable de ce présentateur qui ne cesse de revenir me parler; dans son regard qui m'observe sans me voir, figure hétéronomique de la loi; dans ce monde de l'écran qui me propose de vivre "en temps réel" (direct absolu) ce à quoi je ne puis pourtant accéder, cette actualité loin de moi; dans la mobilisation de ce fantôme du numérique, produit hors de la lumière du jour, qui donne aux images moins le statut d'apparition que de concrétisation d'un univers mentalisé. Promesse de contact et d'impact, le régime présentatif de la télévision est donc un régime phatique, implicatif, où médiations techniques et croyances téléspectatorielles vont de concert.

\subsection{Complexité de l'agir télévisuel}

Longtemps, les professionnels de la télévision française ont pensé et affirmé que l'action globale du média de service public (1950-82 en France) pouvait et devait se circonscrire à la trilogie fonctionnaliste: informer, éduquer, distraire. Cette trilogie revendiquée par la télévision française des origines a donc subsumé les modalités de l'agir téléologique et fondé des habitus englobant leurs éthos respectifs ${ }^{12}$. Le passage à un système officiellement mixte (après 1982) n'a pas débouché ipso facto sur le renversement de ladite trilogie et son remplacement par de nouvelles finalités de référence. Il s'est d'abord limité à la mise en amont de la visée spectaculaire divertissante, glissement dans l'agir spectaculaire et dramaturgique, dont TF1 représente le modèle parfait. Puis, le nécessaire renouvellement des dispositifs d'émissions et l'arrivée de nouvelles réglementations politiques ( loi de 1982) aidant, ont émergé d'autres modalités d'action. Si bien qu'il peut paraître à présent indispensable de repenser la théorie de l'agir communicationnel, de type médiatique et plus spécifiquement télévisuel.

En partant du schéma proposé par A. Gosselin au sujet de la communication politique et en l'adaptant à la communication télévisuelle, j’ai avancé 
l'idée de penser l'agir télévisuel comme stratégie globale d'articulation de plusieurs formes particulières d'agir ${ }^{13}$ ou de rationalités stratégiques en synergie et parfois en conflit. Ces rationalités sont portées par le régime d'action matérielle (finalité commerciale ou marchande) et par le régime d'action symbolique (finalité culturelle au sens large). Dans la première période baptisée par certains "paléo-télévision" (Eco, Odin et Casetti), l'agir télévisuel de type téléologique reposait sur le régime d'action symbolique et les grands types d'imaginaires liés à la parole, à l'identité sociale et à la représentation ${ }^{14} \cdot$ Sous la pression du régime d'action matérielle à visée marchande qui s'est progressivement imposé, la néo-télévision a infléchi son action, sans jamais oublier certaines de ses missions d'origine. Les pratiques habituelles de séparation des genres ont été relayées par des pratiques d'hybridation (psy show, talk show, réality show). L'éthos de l'objectivité, de la neutralité et du sérieux s'est autorisé les valeurs de l'engagement humanitaire, de la solidarité (agir axiologique affirmé) et de la convivialité (prolongement de l'ancienne sociabilité). La spectacularisation, qui se cantonnait jadis au divertissement et à la fiction, a gagné aussi l'espace de l'information et des émissions de plateau. L'agir affectuel qui était relativement timoré et contraint s'exprime à présent sans grande retenue. En somme, la télévision française de la fin du siècle déploie un agir protéiforme. Dans ce qui suit, je vais tenter d'en rendre compte au seul niveau de la construction institutionnelle de la citoyenneté, telle qu'on peut l'appréhender dans les émissions de débat et le régime de la présentation.

\section{Le média télévision et la construction du lien social}

Le régime d'action symbolique de la télévision se présente comme un régime scopique. C'est dire que ce réseau figurant agit par la construction de visibilité-audibilité, intelligibilité, affectivité, dans le cadre d'un espace public de référence.

\subsection{La télévision comme espace public médiatique}

Il ne conviendrait pas de penser l'espace public télévisuel selon les grands modèles historiques de référence: espace public grec de l'agora (Aristote), avec sa distinction public/privé et son esthétique de la présentation de soi; espace public bourgeois (Habermas), avec son institutionnalisation de la critique, son motif moral d'émancipation et son concept normatif d'opinion fondé sur la raison. Avec l'avénement des médias - radio, télévision, presse écrite et édition - l'espace public moderne a connu un double mou- 
vement d'extension et de complexification. Horizontale, l'extension a entrâ̂né l'espace public national à s'internationnaliser. Verticale, elle l'a conduit à transgresser les limites de la privatisation stricte et à se doter de la profondeur d'une mémoire collective. La complexification est actuellement si grande qu'il convient de voir dans la notion trop homogène d'un "espace public médiatique" une pluralité d'espaces pris dans des mouvements "structurants-structurés" et des logiques ou rationalités plus ou moins convergentes.

Certains analystes proposent d'y repérer au moins trois mondes - vie politique, vie civile, vie privée ${ }^{15}$ - et des opérations de glissement perpétuel d'un monde à l'autre. En fait, il semble nécessaire d'aller plus loin encore dans la mise au point du modèle multipolaire et réticulaire qui s'impose.

L'espace public télévisuel du politique est le secteur d'interactions entre différents acteurs, figures institutionnelles représentatives ou citoyens anonymes: personnel politique de l'Etat et des partis, journalistes de tous les médias, instituts de sondage, intellectuels et personnalités (mondes scientifique, économique, artistique, judiciaire,etc), leaders d'opinion éphémères ou durables. Ces acteurs composent des réseaux de relations pouvant varier du système homogène aux structures multipolaires. Ainsi, il a déjà été montré que, jusqu'en 1974 en France, dans le cadre de la télévision de service public de l'ère gaulliste, cet espace se résumait à un "espace public médiatique de l'Etat" ${ }^{\prime 16}$. A cette homogénéité forte a succédé, de 1974 à 1981, une dissociation en espace public de l'Etat - visible notamment lors de la campagne officielle des élections présidentielles - et en espace médiatique de linformation. Bref, la complexification de ce premier espace a entraîné la diminution du poids de l'Etat et la déstabilisation relative de la représentation politique. La domination du principe médiatique sur le principe politique, dont témoignent le règne des sondages, qui vient légitimer le pouvoir des journalistes, et le succès durable des émissions satiriques, où la marionnette du journaliste préside à la mise en dérision du politique, se lit à maints niveaux, dans l'apparition de stratégies médiatiques de captation spectaculaire du politique et de gestion euphémisée de la contradiction. Dans le premier cas, le politique est privatisé (l'émission Questions à domicile), déspécialisé, mêlé à d'autres univers (plateaux de participants issus de tous les domaines), traité de manière humoristique (Les guignols de l'info). Dans le second cas, aux paroles politiques de proclamation ou de déclaration, que les médias se chargent traditionnellement d'extirper et de répercuter, on substitue des contre-propositions venant des gens de la base, de la rue, du commun des mortels ou d'animateurs - présentateurs en mal de mission humanitaire. 
Le monde de la vie sociale qui veut accéder aux écrans de télévision éclate lui aussi en multiples configurations. Celles-ci composent l'espace public télévisuel du social. L'espace public télévisuel ou EPT du littéraire, sans aucun doute initié par B. Pivot dès 1975 (Apostrophes), sait manifester une belle vitalité. L'EPT de l'artistique pointe notamment dans les émissions qui font se côtoyer vedette politique, mannequin, acteur ou actrice ( 7 sur 7 ), timide réactivation de la mythologie cinématographique de la star comme pôle d'identification. L'EPT du scientifique a ses personnalités d'hier - le commandant Cousteau, le vulcanologue H. Tazieff, l'explorateur P-E. Victor (décédé) - et ses célébrités d'aujourd'hui - G. Charpak, G. de Gennes, C. André-Deshayes - qui conjuguent goût de la vulgarisation et acceptation des paillettes. L'EPT du judiciaire propulse ses propres atouts: les juges J. Pierre, R. van Ruymbecke, E. de Mongolfier. Le souci de leur propre légitimation culturelle pousse les professionnels des médias à activer l'EPT du médiatique. Il s'agit de toutes ces émissions où la télévision s'engage dans la visée patrimoniale et valorise ses archives. Ce phénomène est d'abord apparu avec la privatisation de certaines chaînes qui a entraîné notamment la rediffusion d'émissions anciennes et de documents rares. Il s'est consolidé avec la naissance d'un corps de conservateurs des vies et oeuvres médiatiques, dont les noms les plus célèbres sont $\mathrm{P}$. Tchernia et J. Chancel, lesquels se sont autopromus en exégètes, commentateurs, historiens garants des valeurs ${ }^{17}$. L'EPT de la vie civile, quant à lui, a deux visages indissociables: le visage abstrait des sondages construisant l'opinion publique, et la figure tout à fait concrète du citoyen ordinaire, le plus souvent victime des faits divers ou des affaires.

L'espace public télévisuel du privé triomphe dans le psy show, talk show, réality show, qui font de tout individu et de sa vie privée - familiale, sentimentale, conjugale - l'enjeu d'une nouvelle dicibilité et parfois d'une plus forte visibilité. Affirmer sur l'espace public les valeurs de sa personnalité la plus intime permet de contourner les préjugés, tabous et autres normes axiologiques rigoristes ainsi que d'engager la télévision dans une fonction catharrtique exhibée.

Au total, si la télévision demeure l'espace public de référence, il ne faut pas pour autant en conclure que la médiation qui s'y joue est aisément décryptable, ni que le média est, dans le domaine journalistique, le pôle d'où tout part et où tout revient. Le domaine du médiatique est en effet hétérogène. Certaines affaires, politiques ou non, publiques ou privées, peuvent naître dans la presse écrite, dans les livres ou à la radio, être relayées ou non par la télévision, qui servira à l'occasion de caisse de résonance, mais pas forcé- 
ment de révélateur ou de détonateur. Tous les espaces publics télévisuels qui émergent, s'imposent un moment ou glissent dans l'ombre méritent d'être envisagés dans leur double dimension, sociologique - comme pratiques médiatiques à forte composante stratégique - et psychologique comme représentations évocatrices d'imaginaires puissants. Des alliances restreintes à quelques catégories d'espaces - espace médiatique, politique et judiciaire, par exemple; ou espace médiatique et espace privé - se nouent et se dénouent. Et lorsque le régime scopique verse dans la mise en spectacle contagieuse, il le fait pour des raisons qui concernent tout autant le régime d'action matérielle valorisant la marchandise, l'industrie culturelle et l'audimat que le régime d'action symbolique rattaché à des imaginaires personnels et collectifs.

\subsection{Média d'information et citoyenneté}

Se poser la question du rôle de la télévision - espace public de référence configuré en réseau multipolaire de micro-espaces publics à stratégies communes et autonomes à la fois - dans le développement de la citoyenneté, c'est se demander avant tout comment un tel espace médiatique complexe peut produire en son sein un espace qui puisse contribuer à l'édification du citoyen.

Avec J. Derrida, on peut définir la citoyenneté comme "inscription en un lieu, dans un territoire ou dans une nation dont le corps est enraciné dans un territoire privilégié, donné, perdu ou promis" ${ }^{18}$. Tous les termes méritent un examen attentif. La citoyenneté fortifie le lien social, elle dit le rattachement à un pays et à son histoire, elle parle d'enracinement géographique et symbolique, elle est ancrage. Le lieu qu'elle rappelle et exhibe se veut site de vie à expansion infinie: contrée proche, territoire des ancêtres, nation, monde. Ce site physique et symbolique, dont les emblèmes et rituels ne cessent d'être convoqués et mobilisés pour revivifier l'imaginaire, passe par un site télévisuel qui le convertit en espace de référence, selon des médiations fort complexes.

Garants de ladite citoyenneté, la démocratie politique d'une part, comme cadre juridique d'autorisation, et l'institution télévisuelle d'autre part, comme lieu d'effectuation des comportements fondamentaux attachés à la citoyenneté, doivent alors entretenir l'espace civique télévisuel. Et la construction d'une citoyenneté médiatique vaudra peu ou prou comme équivalent d'une citoyenneté sociale. Cet espace civique télévisuel peut alors conjuguer:

- un espace de dicibilité civique où s'expérimentent les droits et les limites de la parole démocratique et du débat contradictoire;

- un espace de visibilité sociale où se montrent / s'exhibent les identités indi- 
viduelles et collectives d'acteurs sociaux (les citoyens) ainsi que les modalités d'ancrage aux sites respectifs de la citoyenneté;

- un espace de réflexivité où la nation se construit la scène de son histoire comme les modes de représentation de son lien social, et s'y regarde en train d'évoluer, entre rapports hiérarchiques de domination et rapports symétriques de coopération; entre production de références identitaires nationales et dépassement de celles-ci dans les formes symboliques de l'universel.

- un lieu d'opérations de médiatisation comme facteur d'opacité, qui nous parle surtout des propriétés du média - télégénisation, légitimation de l'éphémère, spectacularisation, personnalisation - et des stratégies de résistance / acceptation des citoyens présents sur les plateaux.

Du côté de l'instance politique, il s'agit essentiellement de contribuer à la construction et à l'entretien de la notion d'identité, celle de tout citoyen et celle de la nation. Il s'agit par voie de conséquence de faire le même travail sur la notion d'altérité et de relation à l'altérité. Du côté de la télévision, il y a à présenter et représenter de la manière la plus complète les scènes de la citoyenneté, tout en donnant, du moins cela serait souhaitable, les moyens de comprendre les modalités de leur médiatisation. On a souvent relévé que le politique est moins ancré aujourd'hui à ses lieux de prédilection, organes de délibération et de décision - le gouvernement, le Parlement - qu'il n'est tributaire des écrans de télévision. Cette situation engage à une nouvelle construction des concepts d'Etat, de politique et de citoyen, en liaison avec le concept de territoire. Par son régime d'action matérielle, la télévision privée et parfois même certaines chaînes publiques brandissent les impératifs économiques comme autant de limitations de la sphère du politique, jugée vite moins rentable que les jeux et divertissements. En son régime d'action symbolique, la télévision dans son ensemble provoque une certaine dislocation des relations traditionnelles du citoyen aux notions de territoire, nation, frontière, enracinement. Si bien qu'en France, à l'heure actuelle, la question se pose de savoir quel serait le bon niveau de traitement de la référence identitaire: espace public régional, national ou européen, voire mondial? Au niveau local du territoire, la télévision tente d'exprimer les formes de l'échange culturel et social en versant parfois trop dans uns sorte de culture folklorisée, qui est un compromis fade entre désir de proximité et besoin d'universalité. On comprend alors pourquoi certains, comme Dominique Wolton, privilégient le niveau national et la télévision généraliste pour la mise en visibilité d'une identité issue tout droit de l'histoire, facteur de cohésion, creuset d'intégration des différences de tous ordres. Mais cette conception d'un espace 
symbolique national comme protection de la référence identitaire ne serait-elle pas trop timorée? L'expérience d'Arte est certes encore trop balbutiante pour qu'on puisse affirmer la nécessité incontestable d'un espace public supranational, en l'occurrence européen. Cependant, l'effort d'Arte pour exprimer l'identité culturelle de l'Europe, l'unité dans la diversité, l'idée encore abstraite d'un héritage commun, mérite d'être salué. Avec Arte, on saisit ce que représente l'objectif de construction d'une référence identitaire dépassant le niveau national. A cet effet, le média doit être tout à la fois instance de diffusion de la diversité culturelle, instance de médiation qui explicite les valeurs communes et instance de création d'une culture audiovisuelle européenne. Il doit se faire acteur de la formation d'une opinion publique échappant pour partie au seul champ du politique et pensée comme vision du monde attachée au média. Car un espace public supranational exige sans doute qu'à la télévision de médiation s'ajoute une télévision de création.

Si donc une nouvelle éthique, celle de citoyen d'un monde- patrie des droits de l'homme, veut emerger et progresser dans l'espace civique télévisuel, elle doit passer par de nouvelles stratégies de dicibilité, visibilité, intelligibilité. Au plan de la dicibilité, les droits du citoyen ne sont garantis que s'ils ouvrent le plus largement possible aux droits d'intervention discursive sur l'espace public et aux droits de réponse ou droit de suite. Au plan de la visibilité, face à la montée des nationalismes et intégrismes, il peut paraître vital d'ouvrir les écrans de la télévision à la monstration d'expériences, cérémonielles ${ }^{19}$ ou non, de singularités démocratiques, françaises et étrangères. Au plan de l'intelligibilité, seul l'engagement résolu des professionnels des médias dans la réflexivité critique, cette autocritique de leurs pratiques, permettra au citoyen de lutter contre cette forme d'analphabétisme qui frappe les consommateurs d'émissions; de comprendre la nouvelle temporalité de la technique et les risques de dérapages qu' elle recèle ( affaires du charnier de Timisoara, de la fausse interview de Fidel Castro). Or, pour une seule (bonne) émission du type Arrêts sur image, combien d'émissions d'autopromotion qui donnent à penser que la télévision serait un média sourd à toute critique, externe et interne ! Enfin, la citoyenneté passe encore par le droit de consultation des archives ${ }^{20}$, l'exercice de la mémoire étant une condition de compréhension des écrans du présent, et par le droit de suspicion légitime.

\section{Médiatisation de l'échange démocratique}

Les émissions de conversation, débat, talk show ne représentent, il faut le rappeler, qu'une partie des programmes de la télévision. Elles s'inscrivent 
dans le régime de la présentation télévisuelle, gouvernée par les jeux de la dicibilité médiatique et de la monstration de plateaux de paroles en actes. Examiner ces émissions sous l'angle de la citoyenneté et de l'impératif démocratique, c'est étudier en quoi les dispositifs qui les fondent tentent de colmater ou entretenir un véritable déficit symbolique: le déficit d'expression, d'échange et de contradiction.

\subsection{Parole médiatique et ordre du symbolique}

Quand la télévision fait la promotion de la parole cathodique, elle prétend toujours poursuivre une finalité politique, que l'on pourrait appeler son "effet de démocratie". Il s'agit alors de substituer aux limites de la démocratie représentative, qui passe par les porte-parole mandatés, légitimés, élus, tous experts crédibles et fiables en quelque sorte, le mythe de la démocratie directe. A une vision stratifiée et hiérarchisée de la société semble se substituer une conception apparemment égalitariste, qui en appelle -sans détours mais pas sans médiations- aux anonymes devenus témoins et victimes. A la parole institutionnelle de jadis, parfois emprisonnée dans la langue de bois, succède ou prétend succéder la parole vive, libre, sauvage, dont les sites télévisuels électifs sont le plateau-agora et le plateau-confessionnal. Les actes discursifs ainsi déployés ont d'évidentes fonctions catharrtiques, à travers lesquelles les corps individuels et le corps social expurgent les démons des "affaires" et des pathologies. Tend à s'imposer la représentation d'un monde complexe traversé d'événements souvent conflictuels et de cas parfois dramatiques, dont la rationalité peut légitimement échapper. Face à l'élargissement considérable de l'espace public ainsi réalisé, le citoyen peut alors avoir de la peine à construire par réflexion et distanciation ses propres repères, à inscrire ses points de vue dans un schéma d'intelligibilité des phénomènes sociaux.

\subsection{La permanente construction d'identités médiatiques}

En passant du débat au talk show, dans la période de 1960 à 1997, les scènes médiatiques françaises de la conversation ont ouvert, à côté de l'espace originel de la parole légitimée, de la problématisation, de la quête de rationalité et de coopération cognitive, l'espace actuel, éclaté et polymorphe, de la parole "déchaînée". Le goût de la réflexion patiente, de l'argumentation exigeante, de l'analyse rigoureuse de dossiers, se trouve à présent battu en brêche par l'ascension du talk show, où l'agir télévisuel se limite souvent à l'agir dramaturgique et spectaculaire et à l'agir affectuel. La libération de l'indicible, de l'inédit et de l'interdit, parfois véhémente et occasionnellement 
blasphématoire, a fortifié l'attrait de la construction d'identités médiatiques.

Hier, le journaliste présentateur de débat était une sorte de personnage intermédiaire produit par le média, un intellectuel-journaliste au service de la finalité démocratique et culturelle du débat. Primauté du régime cognitif d'intelligibilité! A présent, l'animateur d'émissions de conversation est souvent une sorte d'acteur au milieu de multiples identités. Il s'agit d'abord d'identités stratégiques ${ }^{21}$, construites par les conseillers en communication ou par l'habitude de la représentation institutionnelle; elles concernent alors les hommes politiques, les syndicalistes, les délégués. Il s'agit ensuite d'identités singulières, exhibées par des anonymes dont la personnalité et l'authenticité crèvent l'écran et qui viennent pour s'exprimer, se libérer, porter témoignage. Il s'agit enfin d'identités télégéniques, dont les modes de manifestation se coulent à merveille dans les canons chers au média. Primauté du régime passionnel d'affectivité ! Ainsi, sur la chaîne publique France 2, face à B. Tapie et J-M. Le Pen, Paul Amar ouvre le duel contradictoire en produisant des gants de boxe. Contesté pour ce geste qui le conduit à partir et à gagner la chaîne privée $T F 1$, et face à Maurice Papon en instance de procès pour “crime contre l'humanité", il provoque à nouveau l'événement médiatique en mettant sous le regard de son invité les photos de deux petites filles juives déportées en camp de concentration, ceci dans l'espoir de le déstabiliser et de provoquer l'erreur, en l'occurrence un geste détestable d'humeur et de rejet. Dans les deux cas, le dispositif télévisuel de la conversation entretient la confusion des espaces publics. Un espace politico-médiatique est donné tour à tour pour une salle de boxe ou une salle de tribunal. Et le même présentateur bondit de la quête de savoir à la provocation de l'affect. Royauté de l'agir dramaturgique et spectaculaire!

La démocratie cathodique ne sera jamais comparable à celle des préaux du terrain politique ou à celle de l'agora d'une Assemblée nationale. Elle passe et passera toujours par des opérations de médiation et des modalités de médiatisation, bref par un média qui ne peut qu'exercer une double action, au plan matériel et au plan symbolique. Dans tous les cas, ce phénomène d'intermédiation est facteur de complexification et d'opacité - d'autres parleront de mystification, tromperie ou leurre - qui travaillent l'amont et l'aval du processus de communication. Sur le versant institutionnel, il paraît maintenant nécessaire de ne pas exagérer les réalités de la télécratie, dans la 
tentation d'en faire une véritable iconocratie, ce gouvernement des images et du territoire par les images. Si influence du média sur les téléspectateurs il y a, elle est à penser, pour le sujet qui nous occupe ici, comme contruction complexe d'un espace public de référence, lui-même réseau multipolaire de micro-espaces en interrelations constantes, et lui- même porteur d'un espace civique médiatique. Sur le versant politique, il ne faut pas croire naïvement au mythe de la démocratie directe sur écran de télévision. Mais il est possible, nécessaire et indispensable d'améliorer la valeur civique des émissions, en termes de gains de dicibilité et visibilité, et au prix de la promotion plus courageuse d'une réflexivité critique sans concessions. Si le citoyen doit pouvoir regarder comment se construisent l'identité de chacun et celle du territoire sur la scène télévisuelle de l'Histoire, le journaliste doit aussi pouvoir comprendre comment se construit son identité professionnelle et comment les dispositifs successifs façonnent l'espace civique médiatique où se joue, dans nos sociétés occidentales modernes, l'essentiel du travail démocratique.

\section{Notes}

${ }^{1}$ La notion de médiasphère est avancée par Régis Debray, le fondateur de la médiologie, dans sa tétralogie: Cours de médiologie générale, Gallimard (1991), Vie et mort de l'image, Gallimard (1992), L'Etat séducteur, Gallimard (1993), Manifestes médiologiques, Gallimard (1994).

${ }^{2}$ E. Veron, "Interfaces. Sur la démocratie audiovisuelle avancée”, Hermes 4, CNRS, 1989, $113-126$.

${ }^{3}$ J-M Salaün, "L’aménagement de la culture”, in B. Miège, Médias et communication en Europe, PUG, 1990, 25-42.

${ }^{4}$ La notion est prise dans le sens défini par B.Miège, op cit.

${ }^{5} \mathrm{~N}$. Nel, "Les régimes scopiques" 1 et 2, Champs visuels 1 et 2, mars et juin 1996.

${ }^{6}$ P. Bourdieu, Questions de sociologie, Editions de Minuit, 1984. Le concept d'habitus qui est pour Bourdieu un bon concept permettant d'échapper à l'alternative du mécanisme et du finalisme et de fonder avec plus de pertinence une science des pratiques, signifie un "métier", un capital de techniques et de références, un ensemble de croyances.

Je remercie J. Walter de m'avoir signalé la position de Bourdieu sur la télévision dans les années 1980, ainsi que l'intérêt du modèle d'A. Gosselin (cf note 13).

7 P. Bourdieu, "L'emprise du journalisme”, Actes de la recherche en sciences sociales, 101-102 , mars 1994,5 et 7 .

${ }^{8} \mathrm{~B}$. Miège, "La (nécessaire) voie étroite de la recherche", CinemAction n. ${ }^{\circ}$ 63, Les théories de la communication, 123-129. Au titre des logiques, il cite: la mise en réseau, l'éclatement de l'espace public, la ritualisation sociale, l'industrialisation de l'éducation, de la formation, de l'information et de la culture, la médiatisation croissante de la communication, la mondialisation des marchés publicitaires.

9 D. Chateau, Le bouclier d'Achille. Théorie de l'iconicité, L'Harmattan 1997, 64.

${ }^{10} \mathrm{JP}$ Esquenazi, Le pouvoir d'un média. TF1 et son discours, L'Harmattan, 1996. 
JP Esquenazi, “Qu'est-ce qu'un réalisme ? “et “Qu'est-ce qu’un discours vrai ?”, Champs visuels 1 et 2, mars et juin 1996.

N.Nel, Le débat télévisé, A.Colin, 1990.

${ }^{11}$ J. Derrida et B. Stiegler, Echographies de la télévision. Entretiens filmés, Galilée - INA, 1996.

${ }^{12} \mathrm{P}$. Bourdieu: un habitus est un "système de dispositions acquises par l'apprentissage implicite et explicite qui fonctionne comme un système de schèmes générateurs “, op cit, (119). C'est un systèmes de pratiques et de schèmes de perception des pratiques (135). Un éthos est un "ensemble objectivement systématique de dispositions à dimension éthique, de principes pratiques “(133), ces principes étant logiques, axiologiques, théoriques et pratiques à la fois.

${ }^{13}$ La proposition de A. Gosselin figure dans “La communication politique. Cartographie d'un champ de recherche et d'activités “, Hermès 17-18, 1995, 17-33. La proposition que j’avance est dans “ Généricité, séquentialité, esthétique télévisuelles”, Réseaux, 1997, n. sur les genres télévisuels, à paraître.

${ }^{14}$ P. Charaudeau et R. Ghiglione, La parole confisquée. Un genre télévisuel: le talk show, Dunod, 1997, 27. Les auteurs rattachent constamment les émissions à leurs imaginaires respectifs.

${ }^{15}$ P. Charaudeau et R. Ghiglione, op cit.

${ }^{16}$ E. Veron, op cit, Hermès 4, 122-123.

${ }^{17} \mathrm{P}$. Bourdieu me semble aller un peu vite en besogne quand il déclare que l'apparition d'un corps de conservateurs de vies serait un indice assez clair de la constitution d'un champ. Si cela est vrai dans le secteur de la culture éditoriale, cela l'est moins pour la culture de flot. La quête de légitimité y est toute récente et mal assurée, alors même que la construction d'un espace public remonte aux origines de la télévision de masse. ${ }^{18} \mathrm{~J}$. Derrida et B. Stiegler, op cit, 67-68.

${ }^{19}$ E. Katz, "La recherche en communication depuis Lazarsfeld", Hermès 4. Il signale qu'en certaines occasions - Sadate à Jerusalem, le Pape en Pologne, les funérailles de Kennedy, etc - la télévision unifie le monde. Ces occasions conjuguent la technologie du direct, le caractère extraordinaire de l'événement, la visée communautaire (et parfois festive) de renforcement des valeurs de citoyenneté.

${ }^{20}$ Il faut préciser que la loi française dite "du dépôt légal”, qui autorise dans certaines conditions restreintes le travail des chercheurs sur les archives de télévision,ne date que du 1er janvier 1995 .

${ }^{21}$ J'ai développé plus longuement ce point dans mon article "Le désir d'identité dans la conversation TV", revue L'Image, 1997 (à paraître). 